\title{
A Retrospective Analysis of the Presentation, Outcomes and Determinants of Severity of Postoperative Pneumonia in Upper Abdominal Oncological Surgeries
}

\author{
Prasanna Vani Vanamail, Radhika Dash, Kalpana Balakrishnan \\ Department of Anesthesiology, Cancer Institute (WIA), Chennai, Tamil Nadu, India
}

\section{Abstract}

Context: Postoperative pneumonia (POP) is a major cause of morbidity following upper abdominal oncological surgeries. Aims: To estimate the incidence of POP following upper abdominal oncological surgeries and to identify its clinical and microbiologic features and the risk factors for the development of severe pneumonia. Settings and Design: Retrospective analysis of patients with POP at a regional cancer center. Patients and Methods: Patients' preoperative and intraoperative details and data on clinical and radiological features and pathogens isolated were collected. Patients with severe pneumonia were identified on the basis of Infectious Disease Society of America/American Thoracic Society criteria and their predictors were estimated. The types of respiratory support required and their outcomes were analysed. Statistical Analysis Used: Parametric Student's $t$-independent test was used to compare two groups' means. Categorical data are presented as frequency and percentage values. $P<0.05$ was considered statistically significant. Results: Incidence of POP was $15.24 \%$ with $48 \%$ developing severe pneumonia. There was a significant difference in the mean age ( $59.5 \pm 8.62$ vs. $50.15 \pm 10.53$ years, $P=0.024)$, duration of surgery ( $315.77 \pm 107.29$ vs. $432.92 \pm 177.18 \mathrm{~min}, P=0.055)$, blood loss $(446.15 \pm 260.18 \mathrm{ml}$ vs. $712.5 \pm 326.22 \mathrm{ml})$, time to empiric antibiotic $(15.69 \pm 5.77 \mathrm{vs} .42 \pm 38.12 \mathrm{~h}$, $P=0.022)$, and symptom resolution $\geq 3$ days $(23.1 \%$ vs. $72.7 \%)$ between patients with nonsevere and severe pneumonia. Patients presenting with cough (odds ratio $=0.06,95 \%$ confidence interval: $0.006-0.618$ ) were more likely to have nonsevere pneumonia. Conclusions: Predictors of severe pneumonia are elderly, prolonged surgical duration, higher blood loss, delayed empiric antibiotic and delayed symptom resolution.

Keywords: Cancer surgeries, high-flow oxygen therapy, postoperative pneumonia, upper abdominal surgeries

\section{InTRODUCTION}

Postoperative pneumonia (POP) is considered the second most important predictor of long-term survival following oncological surgeries next only to tumor grade ${ }^{[1]}$ Patients undergoing upper abdominal surgeries for cancer possess a multitude of risk factors predisposing them to POP. ${ }^{[2-5]} \mathrm{POP}$ increases hospitalization and treatment costs. ${ }^{[6,7]}$ POP delays initiation of adjuvant chemotherapy and lowers survival. ${ }^{[8-10]}$ While there are literature aplenty on ventilator-associated pneumonia (VAP), there is limited knowledge on POP. The aim of this study is to estimate the incidence, identify the clinical and microbiologic features of POP and to identify the risk factors for the development of severe pneumonia.

\section{Patients and Methods}

We conducted a retrospective single-center study at a regional cancer center. Data from the hospital records of patients who

\begin{tabular}{|l|l|}
\hline \multicolumn{2}{|c|}{ Access this article online } \\
\hline Quick Response Code: & Website: \\
\hline & www.ijrconline.org \\
\hline
\end{tabular}

underwent elective upper abdominal oncological surgeries over a period of 2 years (April 2017 to March 2019) were analyzed and those who developed POP within 15 days after surgery were included in the study. The investigating team included specialists in anesthesia and intensive care medicine. Upper abdominal oncological surgeries included surgeries for stomach, liver, esophageal and periampullary cancers. We excluded patients with preexisting pulmonary or

Address for correspondence: Dr. Prasanna Vani Vanamail, Room No. 10, Surgical Oncology Block, Cancer Institute (WIA), 38, Sardar Patel Road, Chennai - 600 036, Tamil Nadu, India. E-mail: prasna.vani@gmail.com

This is an open access journal, and articles are distributed under the terms of the Creative Commons Attribution-NonCommercial-ShareAlike 4.0 License, which allows others to remix, tweak, and build upon the work non-commercially, as long as appropriate credit is given and the new creations are licensed under the identical terms.

For reprints contact: reprints@medknow.com

How to cite this article: Vanamail PV, Dash R, Balakrishnan K. A retrospective analysis of the presentation, outcomes and determinants of severity of postoperative pneumonia in upper abdominal oncological surgeries. Indian J Respir Care 2020;9:52-7.

Received: 16-05-2019 Accepted: 14-10-2019 Published: 08-01-2020 
Vanamail, et al:: Postoperative pneumonia in abdominal oncosurgeries

cardiac disease, patients who received elective postoperative ventilation and patients who required rentubation for a nonpulmonary cause. The diagnosis of pneumonia was made in accordance with the Centers for Disease Control and Prevention (CDC) guidelines. ${ }^{[1]]}$ In patients with recurrent episodes of pneumonia, only the first episode was taken into account.

The age, gender, body mass index, comorbidities, preoperative length of stay, nutritional risk screening (NRS 2002), diagnosis, surgery done, duration of surgery, and perioperative blood transfusion details were collected. The time to development of initial symptom following surgery and the time to empiric antibiotic after symptom onset were noted. The Infectious Diseases Society of America (IDSA) major and minor scores were noted for each patient and severe pneumonia was defined by the presence of at least one major criterion or three or more minor criteria. ${ }^{[12]}$ The chest radiographic infiltrates were classified as localized, multiple unilateral, multiple bilateral and diffuse. The culture reports were classified into single or multiple organisms. The antibiotic susceptibility was categorized into penicillin sensitive or resistant for Streptococcus pneumoniae and extended-spectrum beta-lactamase producer or not for Gram-negative aerobic bacteria. The presence of multiple drug-resistant (MDR) pathogens was noted. Appropriateness of the empiric antibiotic was based on the culture reports and in accordance with the antibiotic prescription guidelines. ${ }^{[13]}$ The number of patients who required high-flow oxygen therapy (HFOT), noninvasive ventilation (NIV), endotracheal intubation (ETT), time to intubation from symptom onset, duration of respiratory support, presence of confusion, hypotension and requirement of vasopressors were noted. Systemic bacteremia was considered positive if the patient had positive blood culture with the same organism within 2 days of symptom onset. Delayed intubation was defined as time to intubation $>72 \mathrm{~h}$ after symptom onset. Time to resolution of symptoms was defined by $\mathrm{SpO}_{2}>92 \%$ in room air and respiratory rate $<30 / \mathrm{min}$ without the need for respiratory support for $24 \mathrm{~h}$. The highest Sequential Organ Failure Assessment (SOFA) score during the course of pneumonia was noted. Concomitant intra-abdominal infection was defined as computerized tomography evidence of intra-abdominal collection and organisms isolated from an aseptically obtained culture from the abdominal collection.

\section{Statistical analysis}

Data analysis was carried out using statistical software STATA version 12.0, StataCorp LLC 4905 Lakeway Drive, College Station, Texas 77845-4512,USA. Continuous variables were tested for normality assumptions using the KolmogorovSmirnov test. For normally distributed data, descriptive statistics such as mean, standard deviation and range values were calculated. Parametric Student's $t$-independent test was used to compare two groups' means. For skewed data, median and interquartile range values were calculated. Nonparametric Wilcoxon rank-sum test was used to compare two medians. Categorical data were presented as frequency and percentage values. Unadjusted odds ratio with a $95 \%$ confidence interval $(\mathrm{CI})$ was calculated to assess significant risk variables for the primary outcome. A two-sided probability of $P<0.05$ was considered for statistical significance.

\section{RESULTS}

Of the 165 patients eligible for inclusion, 25 developed POP with an incidence rate of $15.24 \%$. Twelve (48\%) patients had severe pneumonia, of which $66.66 \%$ were male and $33.3 \%$ were female. The types of surgeries included are shown in Figure 1. The incidence of pneumonia and severe pneumonia is shown in Figure 2. Patients undergoing Whipple's surgery had the highest incidence of severe pneumonia (41.6\%), followed by transhiatal esophagectomy (THE; $25 \%$ ) and extended total gastrectomy $(16.6 \%)$.

Hypoxia was the most common presenting symptom seen in $22(88 \%)$ patients, followed by cough in $17(68 \%)$ patients and tachypnea in $14(56 \%)$ patients. Time from symptom onset to starting empiric antibiotic was $<12 \mathrm{~h}$ in $11(44 \%)$ patients, 12 $24 \mathrm{~h}$ in $10(40 \%)$ patients, $24-48 \mathrm{~h}$ in $2(8 \%)$ patients, and $>48 \mathrm{~h}$ in $2(8 \%)$ patients. Three $(12 \%)$ patients had associated systemic bacteremia. Eighteen (72\%) patients had localized infiltrate in the chest X-ray and $6(24 \%)$ patients had multiple bilateral infiltrates. Good-quality sputum sampling was obtained in $19(76 \%)$ patients. Single organism was isolated in $16(64 \%)$ patients and two organisms were isolated in $8(32 \%)$ patients. One patient had negative culture. Streptococcus pneumonia was the most common organism (50\%) isolated in infections with single organism, followed by Klebsiella pneumoniae (25\%) and Pseudomonas aeruginosa (18.75\%). The type of organism isolated in relation to time to onset of pneumonia is shown in Figure 3. Nine (36\%) patients had infection with extended-spectrum beta-lactamase pathogens and $5(20 \%)$ had infection with MDR pathogens. Sixteen $(64 \%)$ patients required respiratory support. Seven (43.75\%) patients were managed with HFOT. Of these, one patient required NIV after 3 days of HFOT and recovered 3 days later. Eight (50\%) patients were started on NIV, of which $4(50 \%)$ required subsequent ETT and 1 (25\%) died due to multiorgan failure. One (6.25\%) patient was directly intubated at onset of respiratory failure due to hemodynamic instability. The time to intubation was $<72 \mathrm{~h}$ in $3(60 \%)$ patients and $>72 \mathrm{~h}$ in $2(40 \%)$ patients. Patients on HFOT required it for a mean period of 2.84 days. Patients on NIV required support for a mean of 2.33 days and patients with ETT required support for a mean period of 2.6 days. Four (16\%) patients received inappropriate empiric antibiotic. The time to symptom resolution was $\leq 48 \mathrm{~h}$ in $9(36 \%)$ patients, $48-72 \mathrm{~h}$ in $6(24 \%)$ patients, and $>72 \mathrm{~h}$ in $6(24 \%)$ patients.

The predictors of severe pneumonia are shown in Tables 1 and 2.

The mean SOFA scores in patients with mild and severe pneumonia were $2.38 \pm 0.65$ and $7.92 \pm 3.655(P<0.0001)$, respectively. 
Vanamail, et al.: Postoperative pneumonia in abdominal oncosurgeries

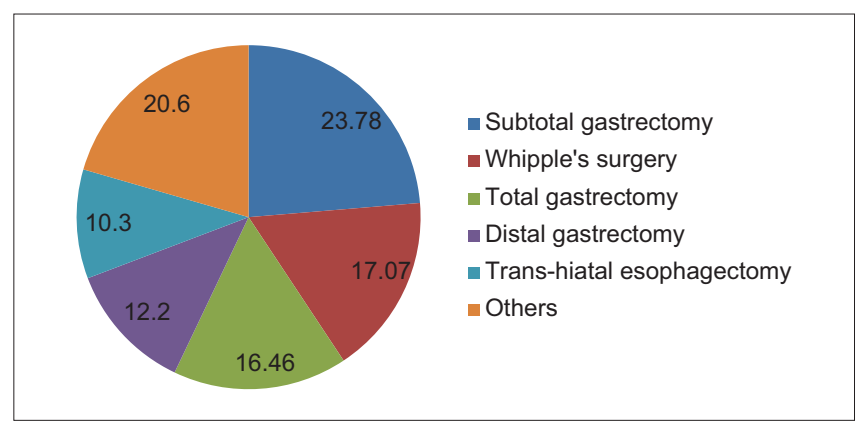

Figure 1: Types of surgeries (\%)

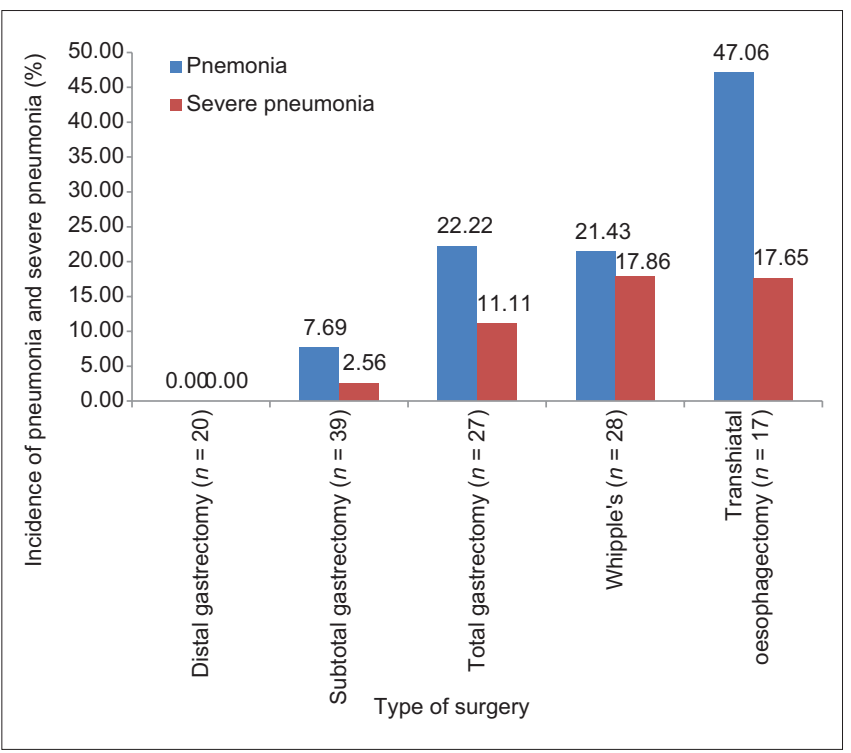

Figure 2: Incidence of pneumonia and severe pneumonia among various surgeries

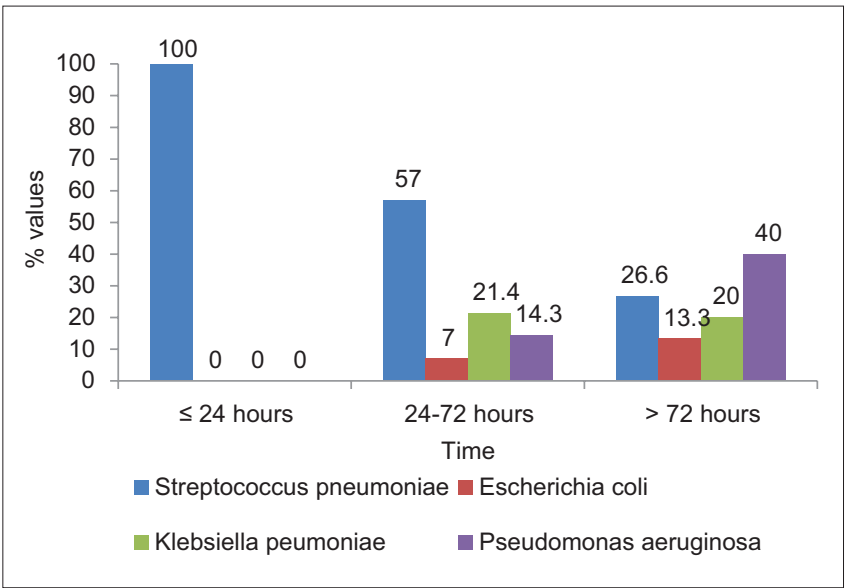

Figure 3: Pathogens isolated in relation to onset of postoperative pneumonia

Four (33.33\%) patients with severe pneumonia had concomitant intra-abdominal infection, of which one patient died.

\section{DisCussion}

There is limited literature on the presentation and outcomes of

\begin{tabular}{lccc}
\hline $\begin{array}{l}\text { Table 1: Predictors of severe pneumonia - Continuous } \\
\text { variables }\end{array}$ & P \\
\cline { 1 - 3 } Parameter & \multicolumn{2}{c}{ Mean \pm SD } & \\
\cline { 2 - 3 } & $\begin{array}{c}\text { Nonsevere } \\
\text { pneumonia }\end{array}$ & $\begin{array}{c}\text { Severe } \\
\text { pneumonia }\end{array}$ \\
\hline Age (years) & $50.15 \pm 10.53$ & $59.5 \pm 8.62$ & 0.024 \\
Hemoglobin (g/dL) & $11.15 \pm 1.69$ & $10.63 \pm 2.43$ & 0.537 \\
Nutritional risk screening & $4 \pm 0.91$ & $3.92 \pm 0.99$ & 0.829 \\
score & & & \\
Charlson comorbidity index & $2.69 \pm 0.95$ & $3.42 \pm 1.38$ & 0.137 \\
Smoking (pack years) & $3.85 \pm 6.50$ & $1.67 \pm 3.89$ & 0.325 \\
Time since smoking & $0.87 \pm 2.75$ & $0.02 \pm 0.05$ & 0.299 \\
cessation (years) & & & \\
$\begin{array}{l}\text { Duration of surgery (min) } \\
\text { Intraoperative blood }\end{array}$ & $315.77 \pm 107.29$ & $432.92 \pm 177.18$ & 0.055 \\
loss (ml) & $446.15 \pm 260.18$ & $712.5 \pm 326.22$ & 0.033 \\
Onset of symptoms (days) & $2.77 \pm 1.74$ & $3.33 \pm 3.39$ & 0.602 \\
Time to empiric & $15.69 \pm 5.77$ & $42 \pm 38.12$ & 0.022 \\
antibiotics (h) & & & \\
Postoperative urea (mg/dL) & $22.46 \pm 6.35$ & $34 \pm 19.24$ & 0.052 \\
\hline SD: Standard deviation & & &
\end{tabular}

\section{Table 2: Predictors of severe pneumonia - discrete} variables

\begin{tabular}{|c|c|c|c|}
\hline Parameter & $\begin{array}{c}\text { Incidence in } \\
\text { nonsevere } \\
\text { pneumonia, } \boldsymbol{n}(\%)\end{array}$ & $\begin{array}{c}\text { Incidence } \\
\text { in severe } \\
\text { pneumonia, } n(\%)\end{array}$ & $P$ \\
\hline $\begin{array}{l}\text { Cough as initial } \\
\text { presentation }\end{array}$ & $12(92.3)$ & $5(41.7)$ & 0.011 \\
\hline $\begin{array}{l}\text { No P/F improvement } \\
\text { at } 72 \mathrm{~h}\end{array}$ & $1(7.7)$ & $6(50)$ & 0.030 \\
\hline $\begin{array}{l}\text { Patients with symptom } \\
\text { resolution } \geq 3 \text { days }\end{array}$ & $3(23.1)$ & $8(72.7)$ & 0.038 \\
\hline $\begin{array}{l}\text { Multiple bilateral chest } \\
\text { X-ray infiltrates }\end{array}$ & $4(30.8)$ & $2(16.7)$ & 0.645 \\
\hline Multiple organisms & $3(23.1)$ & $5(45.5)$ & 0.390 \\
\hline ESBL organisms & $5(38.5)$ & $8(66.7)$ & 0.238 \\
\hline MDR organisms & $1(7.7)$ & $3(25)$ & 0.322 \\
\hline $\begin{array}{l}\text { Inappropriate empiric } \\
\text { antibiotic }\end{array}$ & $1(7.7)$ & $3(25)$ & 0.322 \\
\hline
\end{tabular}

POP in upper gastrointestinal oncological surgeries. Knowledge on the presentation, causative organisms, severity prediction, management modalities and outcomes will help in allocation of appropriate resources in limited availability settings.

Postoperative oncological patients are at increased risk of pneumonia due to multitude of predisposing factors which increase the exposure to hospital-acquired pathogens, impairment in immunity and surgical stress.

Data are limited on the incidence of HAP excluding VAP. In a retrospective study of 1053 gastrectomy patients, the incidence of POP was $2.2 \%$ with nil mortality. Fifty-five percentage of these patients underwent laparoscopic gastrectomy, 5.3\% had proximal gastrectomy and $72 \%$ had distal gastrectomy. ${ }^{[4]}$ In 
Vanamail, et al:: Postoperative pneumonia in abdominal oncosurgeries

our study, all patients had open surgeries with $17.68 \%$ (29) proximal gastrectomy, $10.37 \%$ (17) THE and 35.37\% (58) distal gastrectomy. This could explain the $15.24 \%$ incidence of POP in our study. We found a higher incidence of pneumonia in general and severe pneumonia, in particular in patients undergoing Whipple's surgery and THE. Prolonged duration of these surgeries, constant diaphragmatic retraction and risk of recurrent laryngeal nerve injury predisposing to aspiration could be the putative factors.

We followed the CDC definition of POP in accordance with the recommendation by the core outcome measures in perioperative and anaesthetic care (COMPAC) group. ${ }^{[10]}$ The IDSA/American Thoracic Society criteria was used severity assessment. Intensive care unit (ICU)-level care is needed in the presence of one or more major criteria. The presence of three or more minor criteria indicates severe pneumonia and the likely need of ICU-level care. ${ }^{[12,14]}$

Among the presenting features, hypoxia was the most common (88\%), which could be attributed to atelectasis and diaphragmatic splinting. Fever was seen less commonly in $44 \%$ of patients underscoring the need for high index of suspicion to identify POP. In patients with severe pneumonia, it was found that cough was the presenting symptom in $41.7 \%$ of patients, whereas it was seen in $92.3 \%$ of patients with nonsevere POP $(P=0.011$, odds ratio $[\mathrm{OR}]=0.06,95 \% \mathrm{CI}$ : 0.006-0.618). This could be due to early identification and treatment of POP in patients presenting with cough and hence avoiding a severe pneumonia.

The sampling method predominantly used was sputum cultures in $76 \%$ of patients. In nonventilated patients with ICU-acquired pneumonia, it was found that spontaneous sputum cultures had $82 \%$ sensitivity and $94 \%$ specificity. ${ }^{[15]}$ In a retrospective study comparing the sample of patients who underwent broncho-alveolar lavage (BAL) and sputum collection, it was found that the rate of concurrent positive cultures in paired specimens obtained within 1 or 7 days was $93.7 \%$ and $96.5 \%$, respectively. BAL may be helpful in isolating organisms missed by sputum cultures. ${ }^{[16]}$ According to British Thoracic Society (BTS), bronchoscopy may be considered for patients with nonresolving or slowly resolving pneumonia if they are current or ex-smokers and $>50$ years of age. Its role early in the course of pneumonia is limited. ${ }^{[17]}$

Four patients received inappropriate empiric antibiotic and three of them had severe pneumonia. However, there was no statistically significant difference in severity of pneumonia and appropriateness of antibiotic. This could be due to the small sample size of our study. The main pathogens isolated in patients with inappropriate antibiotic were multidrug-resistant (MDR) $K$. pneumoniae, followed by Escherichia coli and P. aeruginosa. P. aeruginosa and methicillin-resistant Staphylococcus aureus (MRSA) are the most common cause of initial inappropriate empiric antibiotic in patients undergoing intra-abdominal surgeries. ${ }^{[18]}$
Incidence of nosocomial pneumonia was $28 \%$ in patients with intra-abdominal infection with a mortality rate of $66 \% .{ }^{[19]}$ In our study, 33.33\% (4) of patients with severe pneumonia had concomitant intra-abdominal infection and mortality was $25 \%$ (1). In experimental studies, the presence of intra-abdominal abscess is known to decrease the ability of pulmonary macrophages to clear the bacteria. Prolonged antibiotic treatment and hospitalization would favor the development of high-risk pathogens causing pneumonia in these patients. ${ }^{[19]}$ Concomitant intra-abdominal abscess, prolonged duration of surgery $>400 \mathrm{~min}$, isolation of MRSA, and inadequate initial antibiotic therapy were determinants of death. ${ }^{[18]}$

There was a significant difference in the mean age of patients with severe and nonsevere pneumonia, $59.5 \pm 8.62$ versus $50.15 \pm 10.53$ years, $P=0.024$. Pneumonias differ in their presentation due to modified host response in elderly patients. They are also functionally dependent, immunocompromised, and have impaired airway reflexes predisposing them to severe disease. ${ }^{[20]}$

The duration of surgery between patients with nonsevere and severe pneumonia was $315.77 \pm 107.29$ and $432.92 \pm 177.18 \mathrm{~min}$, respectively, $P=0.055$. Prolonged duration of anesthesia and surgery are independent predictors of death from pneumonia due to suppression of cell-mediated immunity by the surgical stress. ${ }^{[21]}$

Six $(37.5 \%)$ patients were successfully managed on HFOT. One patient required subsequent NIV after 3 days of HFOT due to worsening $\mathrm{PaO}_{2} / \mathrm{FiO}_{2}(\mathrm{P} / \mathrm{F})$ ratios and subsequently recovered after 3 days on NIV. In a retrospective review of Pneumocystis carinii pneumonia treated with HFOT, it was found that $\mathrm{P} / \mathrm{F}$ ratios significantly improved in HFOT as compared to mechanical ventilation group. $\mathrm{PaO}_{2}$ improved in survivors as compared to nonsurvivors within $3 \mathrm{~h}$ of initiation of HFOT. Less than 57\% increase in baseline $\mathrm{PaO}_{2}$ had a sensitivity of $87 \%$ in predicting mortality. The 60 -day mortality was $52 \%$ in the high-flow and subsequent mechanical ventilation group, $13 \%$ in the high-flow group, and $30 \%$ in the mechanical ventilation group. ${ }^{[22]}$ The use of HFOT in acute respiratory failure helps in rapid amelioration of dyspnea, improves hypoxemia, improves management of respiratory secretions, and lowers the need for mechanical ventilation. The early predictors of failure of HFOT are persistence of tachypnea, thoracoabdominal discordance, and lack of improvement in oxygenation. ${ }^{[23]}$ Intubation rates did not differ between HFOT, standard oxygen, and NIV group. The 90-day mortality was significantly less in HFOT. The incidence of septic shock was also higher in the NIV group as compared to the HFOT group (30.9\% vs. $17.9 \%$ ), and this could explain the improved 90-day mortality in the HFOT group. ${ }^{[24]}$ On comparing patients intubated early $(<48 \mathrm{~h})$ and late $(>48 \mathrm{~h})$ after HFOT due to lack of improvement, it was found that the mortality was less in patients intubated early. The possible explanation could be delay in diagnosis due to lack of 
definitive airway access, inadequate clearance of secretions and uncontrolled lung stretch contributing to lung injury. ${ }^{[25]}$ Hence, HFOT is of great worth and applicability in the management of hypoxia in pneumonia, but it needs continuous vigilance to assess nonimprovement and need for mechanical ventilation.

There was a significant difference in time to starting empiric antibiotic after symptom onset among patients with nonsevere and severe pneumonia, $15.69 \pm 5.77 \mathrm{~h}$ versus $42 \pm 38.12 \mathrm{~h}$, $P=0.022$. Delay in starting empiric antibiotic could be due to atypical presentation such as altered sensorium and incorrect attribution of hypoxia to atelectasis or other noninfective causes. Patients presenting with cough $(\mathrm{OR}=0.06,95 \% \mathrm{CI}$ : 0.006-0.618) were more likely to have nonsevere pneumonia due to early treatment. In a study on HAP and VAP, it was observed that patients receiving antibiotics within $24 \mathrm{~h}$ after a culture had a significantly shortened length of stay in ICU, 5.62 days compared to those receiving antibiotics at 24-48 h (9 days) and $>48 \mathrm{~h}$ (15.8 days), $P<0.001$. $^{[26]}$

Patients with severe pneumonia had a higher mean intraoperative blood loss, $712.5 \pm 326.221 \mathrm{ml}$ versus $446.15 \pm 260.177$, $P=0.033$. This could be due to prolonged duration of surgery or extensive dissections. Large-volume blood loss can predispose to prolonged systemic hypoperfusion which can activate the stress response mechanism and enhance the catabolic effects of inflammation. ${ }^{[27]}$ Blood loss can cause direct immune dysfunction due to significant loss of leukocytes and can further suppress cell-mediated immunity through depression of T-lymphocytes, macrophage antigen presentation

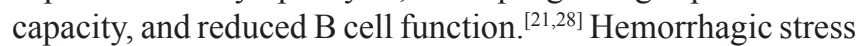
response mediated by antidiuretic hormone-aldosterone and renin angiotensin systems (ADH-aldosterone and RAS) system enhance retention of fluids given for managing hypovolemia and by increasing alveolar capillary hydrostatic pressure and facilitating abnormal capillary permeability can lead to alveolar dysfunction. ${ }^{[2]}$ These factors work in unison in the pathogenesis of POP.

Patients with severe pneumonia had a lack of improvement in $\mathrm{P} / \mathrm{F}$ ratio at $72 \mathrm{~h}$ in $50 \%$ as compared to $7.7 \%$ in nonsevere pneumonia, $P=0.030$. The time to symptom resolution was $>3$ days in $72.7 \%$ of patients with severe pneumonia as compared to $23.1 \%$ in patients with non-severe pneumonia, $P=0.038$. Due to low $\mathrm{P} / \mathrm{F}$ ratios, there was a need to supplement oxygen therapy for prolonged period in patients with severe pneumonia. Worsening $\mathrm{P} / \mathrm{F}$ could be due to filling up of the alveoli with inflammatory exudate compromising the functional residual capacity and due to reduced surfactant activity. ${ }^{[30]}$

Perioperative blood transfusion, preoperative hemoglobin, malnutrition as assessed by NRS, comorbidities, duration, and time since cessation of smoking were not significant predictors of severe pneumonia despite being known risk factors for the development of pneumonia. The limitations of this study are the limited sample size, as we chose to focus on a relatively high-risk population for the development of POP and its retrospective design.

\section{Conclusions}

One in two patients developing POP following upper abdominal oncological surgeries is likely to have severe pneumonia with the need of respiratory or hemodynamic support. Predictors of severe pneumonia are elderly, prolonged surgical duration, higher intraoperative blood loss, delay in starting empiric antibiotic, lack of improvement in oxygenation within $72 \mathrm{~h}$, and time to symptom resolution more than 3 days. HFOT is a valuable tool in the armamentarium of POP, but it needs vigilance in identifying worsening pneumonia and the need for mechanical ventilation when required.

\section{Financial support and sponsorship}

Nil.

\section{Conflicts of interest}

There are no conflicts of interest.

\section{RefEREnCES}

1. Kinugasa S, Tachibana M, Yoshimura H, Ueda S, Fujii T, Dhar DK, et al. Postoperative pulmonary complications are associated with worse short- and long-term outcomes after extended esophagectomy. J Surg Oncol 2004;88:71-7.

2. Smetana GW, Pfeifer KJ, Slawski BA, Jaffer AK, Dutta S, Cohn SL. Risk factors for postoperative pulmonary complications: An update of the literature. Hosp Pract (1995) 2014;42:126-31.

3. McAlister FA, Bertsch K, Man J, Bradley J, Jacka M. Incidence of and risk factors for pulmonary complications after nonthoracic surgery. Am J Respir Crit Care Med 2005;171:514-7.

4. Inokuchi M, Kojima K, Kato K, Sugita H, Sugihara K. Risk factors for post-operative pulmonary complications after gastrectomy for gastric cancer. Surg Infect (Larchmt) 2014;15:314-21.

5. Shirinzadeh A, Talebi Y. Pulmonary complications due to esophagectomy. J Cardiovasc Thorac Res 2011;3:93-6.

6. Kodra N, Shpata V, Ohri I. Risk factors for postoperative pulmonary complications after abdominal surgery. Open Access Maced J Med Sci 2016;4:259-63.

7. Dimick JB, Chen SL, Taheri PA, Henderson WG, Khuri SF, Campbell DA Jr, et al. Hospital costs associated with surgical complications: A report from the private-sector national surgical quality improvement program. J Am Coll Surg 2004;199:531-7.

8. Jin LX, Sanford DE, Squires MH $3^{\text {rd }}$, Moses LE, Yan Y, Poultsides GA, et al. Interaction of postoperative morbidity and receipt of adjuvant therapy on long-term survival after resection for gastric adenocarcinoma: Results from the U.S. Gastric cancer collaborative. Ann Surg Oncol 2016;23:2398-408.

9. Tu RH, Lin JX, Li P, Xie JW, Wang JB, Lu J, et al. Prognostic significance of postoperative pneumonia after curative resection for patients with gastric cancer. Cancer Med 2017;6:2757-65.

10. Trinh VQ, Ravi P, Abd-El-Barr AE, Jhaveri JK, Gervais MK, Meyer CP, et al. Pneumonia after major cancer surgery: Temporal trends and patterns of care. Can Respir J 2016;2016:6019416. doi:10.1155/2016/6019416.

11. Abbott TEF, Fowler AJ, Pelosi P, Gama de Abreu M, Møller AM, Canet J, et al. A systematic review and consensus definitions for standardised end-points in perioperative medicine: Pulmonary complications. Br J Anaesth 2018;120:1066-79.

12. Brown SM, Jones BE, Jephson AR, Dean NC, Infectious Disease Society of America/American Thoracic Society 2007. Validation of the infectious disease society of america/American thoracic society 2007 guidelines for severe community-acquired pneumonia. Crit Care Med 2009;37:3010-6.

13. Khilnani GC, Zirpe K, Hadda V, Mehta Y, Madan K, Kulkarni A, et al. Guidelines for antibiotic prescription in intensive care unit. Indian J Crit Care Med 2019;23:S1-63.

14. Phua J, See KC, Chan YH, Widjaja LS, Aung NW, Ngerng WJ, et al. 
Validation and clinical implications of the IDSA/ATS minor criteria for severe community-acquired pneumonia. Thorax 2009;64:598-603.

15. Stéphan F, Zarrouki Y, Mougeot C, Imbert A, Kortchinsky T, Pilorge C, et al. Non-ventilator ICU-acquired pneumonia after cardiothoracic surgery: Accuracy of diagnostic tools and outcomes. Respir Care 2016;61:324-32.

16. Dubourg G, Abat C, Rolain JM, Raoult D. Correlation between sputum and bronchoalveolar lavage fluid cultures. J Clin Microbiol 2015;53:994-6.

17. Du Rand IA, Blaikley J, Booton R, Chaudhuri N, Gupta V, Khalid S, et al. British thoracic society guideline for diagnostic flexible bronchoscopy in adults: Accredited by NICE. Thorax 2013;68 Suppl 1:i1-44.

18. Fujita $T$, Ishida $Y$, Yanaga K. Impact of appropriateness of initial antibiotic therapy on outcome of postoperative pneumonia. Langenbecks Arch Surg 2008;393:487-91.

19. Reddy RC, Chen GH, Newstead MW, Moore T, Zeng X, Tateda K, et al. Alveolar macrophage deactivation in murine septic peritonitis: Role of interleukin 10. Infect Immun 2001;69:1394-401.

20. Janssens JP, Krause KH. Pneumonia in the very old. Lancet Infect Dis 2004;4:112-24.

21. Angele MK, Chaudry IH. Surgical trauma and immunosuppression: Pathophysiology and potential immunomodulatory approaches. Langenbecks Arch Surg 2005;390:333-41.

22. Kim WY, Sung H, Hong SB, Lim CM, Koh Y, Lyu J, et al. Outcome of high-flow nasal cannula for acute hypoxemic respiratory failure due to pneumocystis pneumonia. In: 2.2 Noninvasive Ventilatory Support.
Vol 48. London, UK: European Respiratory Society; 2016. p. 3052.

23. Sztrymf B, Messika J, Bertrand F, Hurel D, Leon R, Dreyfuss D, et al. Beneficial effects of humidified high flow nasal oxygen in critical care patients: A prospective pilot study. Intensive Care Med 2011;37:1780-6.

24. Frat JP, Thille AW, Mercat A, Girault C, Ragot S, Perbet S, et al. High-flow oxygen through nasal cannula in acute hypoxemic respiratory failure. N Engl J Med 2015;372:2185-96.

25. Kang BJ, Koh Y, Lim CM, Huh JW, Baek S, Han M, et al. Failure of high-flow nasal cannula therapy may delay intubation and increase mortality. Intensive Care Med 2015;41:623-32.

26. Khan RA, Bakry MM, Islahudin F. Appropriate antibiotic administration in critically ill patients with pneumonia. Indian J Pharm Sci 2015;77:299-305.

27. Cué JI, Peyton JC, Malangoni MA. Does blood transfusion or hemorrhagic shock induce immunosuppression? J Trauma 1992;32:613-7.

28. Mizuno A, Kanda M, Kobayashi D, Tanaka C, Iwata N, Yamada S, et al. Adverse effects of intraoperative blood loss on long-term outcomes after curative gastrectomy of patients with stage II/III gastric cancer. Dig Surg 2016;33:121-8.

29. Adeniji K, Steel AC. The pathophysiology of perioperative lung injury. Anesthesiol Clin 2012;30:573-90.

30. Han S, Mallampalli RK. The role of surfactant in lung disease and host defense against pulmonary infections. Ann Am Thorac Soc 2015;12:765-74. 